\title{
MODELLING AND VALIDATION OF THE VEHICLE LONGITUDINAL MODEL
}

\author{
F. Ahmad ${ }^{1, *}$, S. A. Mazlan ${ }^{1}$, H. Zamzuri ${ }^{1}$, H. Jamaluddin', \\ K. Hudha ${ }^{3}$ and M. Short ${ }^{4}$ \\ ${ }^{1}$ Vehicle System Engineering Research Laboratory \\ Malaysia-Japan International Institute of Technology, \\ Universiti Teknologi Malaysia [1], 54100 Jalan Semarak, Kuala Lumpur, Malaysia \\ *E-mail: fauzi.ahmad@utem.edu.my \\ ${ }^{2}$ Faculty of Mechanical Engineering, Universiti Teknologi Malaysia, \\ 81310 UTM Skudai, Johor, Malaysia \\ ${ }^{3}$ Department of Mechanical Engineering, Faculty of Engineering, \\ Universiti Pertahanan Nasional Malaysia (UPNM), Kem Sungai Besi, 57000 \\ Kuala Lumpur, Malaysia. \\ ${ }^{4}$ School of Science and Engineering, Teesside University, Middlesbrough, Tees Valley, \\ TS1 3BA, United Kingdom
}

\begin{abstract}
This paper presents the detailed derivation and validation of a full vehicle model to study the behaviour of vehicle dynamics in the longitudinal direction. The model consists of handling and tyre subsystems, an engine model subsystem, an automatic transmission subsystem and a brake model subsystem. The full vehicle model was then validated using an instrumented experimental vehicle based on the driver input from brake and throttle pedals. Vehicle transient handling dynamic tests known as sudden braking tests were performed for the purpose of validation. Several behaviours of the vehicle dynamics were observed during braking and throttling manoeuvres, such as body longitudinal velocity, wheel linear velocity and tyre longitudinal slip, at each quarter of the vehicle. Comparisons of the experimental results and model responses with sudden braking and throttling imposed motions were made in this study. It is concluded that the trends between simulation results and experimental data were found to be almost similar with an acceptable level of error for the application at hand.
\end{abstract}

Keywords: Modelling; validation; 6-DOF vehicle longitudinal model; Simulation; Experiment.

\section{INTRODUCTION}

As vehicles have become more sophisticated (and hence more expensive), such intuitive development has become very expensive and also risky business [2]. In an effort to predict and quantify the effects of proposed changes in vehicle parameters, designers are increasingly turning to computer simulation techniques to evaluate design proposals. However, computer simulations can only be useful if the software accurately reflects the behaviour of the actual vehicle. If it does, then considerable savings in time and costs can be obtained. Vehicle dynamics models can be developed for simulation using two possible approaches. The first approach uses a multi-body method to generate the equations of motion, where the vehicle is described as a collection of rigid bodies 
connected by appropriate joints and internal forces and subject to external forces [3]. The equations are automatically generated and solved by software packages such as DADS [4], AutoSim or ADAMS [5]. The second approach to vehicle dynamic modelling is known as simplified modelling. There are three main types of simplified vehicle model often used in vehicle dynamics analysis, namely the quarter car, half car and full car models. In the quarter car model, only up-down movements of the sprung and unsprung masses are assumed to take place and the role of the control arm is completely ignored [6]. Meanwhile, the half car model consists of a combination of two quarter-car models, which include the rotational effects of pitch or roll as well as bounce in sprung mass motions. Lastly, the full vehicle model can be divided into a ride model (to simulate a road bump test) and a handling model (to simulate vehicle cornering and/or braking behaviour). Generally, vehicle dynamics analysis using ride and handling are investigated separately and it is assumed that there is no interaction between the two situations. This is due to the assumption in ride model that the input is only from the road bump and there is no driver input from the steering wheel or brake pedal. On the other hand, for the handling model, it is assumed that the vehicle is travelling on a flat road during cornering or braking. In any case, in the development of vehicle models these assumptions should be validated using a real vehicle in order to produce an appropriate vehicle model which can represent a real vehicle. According to Hudha [6], a vehicle model is considered impractical until the model is fully validated using real vehicle data.

In recent years, there has been much research work done on designing and modelling a vehicle, varying the number of degrees of freedom (DOF) and the final purpose of the model. Since validation incurs high costs and extensive efforts, not many researchers have fully validated their models experimentally. As an example, Aparow et al. [7] and Imaduddin et al. [8] have developed a quarter vehicle model and validated the model using a quarter car test rig only. It cannot be assumed that the vehicle dynamic performance in real situations could be the same, even if the result of the validation between the model and the test rig is similar. This is because the behaviour of the quarter car in a real vehicle is influenced by the roll bar/chassis that connects one quarter to the other quarters. Additionally, Ekoru and Pedro [9] developed a 4-DOF half-car model, and Hudha [6] developed a 7-DOF vehicle model using Matlab Simulink Software without validation. Other than that, the software-based validation of a 14-DOF full-car vehicle dynamic model has been reported by Hudha et al. [10]. The model was validated using the Carsim Software. Although the results of the validation are good, the dynamic behaviour of the model representing a real vehicle still needs to be shown. Meanwhile, Hudha et al. [10] and Kadir et al. [11] have presented a simplified 14-DOF full vehicle model and carried out a successful validation using a real vehicle. However these studies only considered the lateral dynamics of the vehicle and evaluated the suspension effects, but neglected the braking performance. In terms of the vehicle longitudinal model, Ahmad et al. [2] have reported that they have successfully validated their 14-DOF full-vehicle longitudinal model with a real vehicle. The intention of this model is to validate translational and rotational motion in a vehicle such as longitudinal acceleration, pitch angle and longitudinal slip of the tyre. Other than that, Hudha [6], El Majdoub et al. [12], Short and Pont [13], they used another method in modelling a longitudinal model. The longitudinal model that was developed was based on a traction model to evaluate the behaviour of longitudinal speed, wheel speed and longitudinal slip, but the models that have been developed have not been validated experimentally. Recently, Short et al. [14] have also developed a vehicle 
longitudinal model based on payload parameter estimator (PPE) and validated it with a real vehicle. Even though the results show a good similarity with the experimental data, these researchers have neglected to include a detailed engine model.

In this study, a handling model is used and derived based upon the longitudinal model proposed by Huang and Wang [15] with the addition of detailed engine dynamics. A 6-DOF model is principally developed to predict the behaviour of a vehicle in a longitudinal direction, and as such the steering effects, yaw angle and lateral acceleration have been neglected. Hudha [6] has commented that "the model is useless until it is validated", in this paper extensive validation tests are carried out with a real vehicle. The experimental vehicle which is employed in this study is a Malaysian national car, namely a Proton Iswara with a 1.3-litre engine capacity and a five-gear manual transmission drive train. The purpose of validation is to determine whether the model is valid enough for its purpose over the complete domain of its intended applicability.

\section{MODELLING METHOD}

In order to simulate the dynamics of a vehicle in longitudinal directions, a 6-DOF passenger vehicle model is considered such as that shown in Figure 1. The proposed full-vehicle model is partially derived from Huang and Wang [15], Bakker et al. [16], which consisted of a single sprung mass (vehicle body) connected to four unsprung masses (wheels). In this study, the sprung mass is represented as a single plane model and is allowed to pitch as well as to displace in longitudinal direction. Each wheel is also allowed to rotate along its axis since the vehicle is driven by two wheels on the front axle. Powertrain model and brake dynamics are included in this modelling as they contribute significantly in developing the vehicle model. Several assumptions have been considered to allow the simulation of the vehicle made in the Matlab Simulink Software as stated in Hudha [6] and Short et al. [14].

\section{Four-wheel Traction Model}

The major elements for the longitudinal vehicle model are the vehicle dynamics and the power train dynamics. The vehicle dynamics are influenced by longitudinal tyre forces, aerodynamic drag forces, rolling resistance forces and gravitational force, while the longitudinal power train system of the vehicle consists of the internal combustion engine, transmission and wheel dynamics. Since the intention of the modelling is to observe the capability of the model to produce the realistic behaviour at each wheel of the vehicle during braking and throttling, a four-wheel dynamic model is needed. Consider a vehicle moving on an inclined road, as shown in Figure 1. The external forces acting on the vehicle include aerodynamic drag forces, gravitational forces, longitudinal tyre forces and rolling resistances forces. These forces and their nomenclatures are described as follows: $m$ is the vehicle lumped mass, $C G$ is the centre of gravity, $L$ is the vehicle wheel base, $B$ is the distance of the front axle to $C G, C$ is the distance of the rear axle to $C G, H$ is the height $C G$ from ground, $\theta$ is the road inclination, $V$ is the body linear velocity acting in longitudinal direction, $\omega_{i j}$ is the wheel angular velocity, $R_{i j}$ is the wheel rolling radius, $J_{i j}$ is the wheel moment of inertia and $\mu_{i j}$ is the road coefficient of friction. The $i$ and $j$ in the nomenclatures are denoted as front or rear and right or left respectively. 




Figure 1. Two-dimensional schematic diagram of the four-wheel traction model.

\section{Vehicle Equation of Motion}

By using Newton's second law, which states that the acceleration of a body is directly proportional to the forces acting on that body multiplied by its mass, the equations of motion can be obtained. First, all the forces that are acting upon the vehicle are divided into two sets of forces: i) the forces acting in the direction of a movement, $F_{x}$, which are applied to the wheels of the vehicle, and ii) $F_{t r}$ the friction forces that are acting in the opposite direction of the vehicle movement. Generally Newton's law can actually be described mathematically as

$$
\frac{d V}{d t}=\frac{\sum F_{x}-\sum F_{t r}}{M}
$$

Since the forces acting on the vehicle are due to the contact of the wheel with the road, the moment balance of the wheel can be described mathematically as Eq.(1), where $\omega_{i j}, J_{i j}$ and $R_{f j}$ are the angular velocity, moment inertia and rolling radius of the wheels, respectively, while $T_{b i j}$ is the applied braking torque, $T_{a i j}$ is the applied throttling torque, $F_{x i j}$ is the longitudinal force acting on the tyres, and $F_{z i j}$ is the force that acting normal to the tyres. The relation between the longitudinal tyre forces and the normal tyre forces is represented as $F_{x i j}=\mu_{i j} F_{z i j}$. The normal forces of the front and rear wheels can be calculated as Eq.(2).

$$
\begin{gathered}
J_{i j} \omega_{r j}=F_{x i j} R_{\omega i j}-T_{b i j}+T_{a i j}+T_{d i j} \omega_{i j} \\
F_{z i j}=m g\left[\frac{\frac{B}{C}}{L} \cos (\theta)-\frac{H}{L} \sin (\theta)\right]+m\left(\frac{d V}{d t}\right) \frac{H}{L}
\end{gathered}
$$

From Eq.(1) and Eq.(2), it can be noted that the summation of the reactions occurring in both the front and rear contact points is the total force acting on the vehicle body which is:

$$
F_{x \text { Total }}=2 F_{x i j}+2 F_{z i j}
$$


The main dynamic states to be simulated in this modelling are tyre rotational velocities for each wheel, $\omega_{i j}$, and the vehicle longitudinal velocity, $V$. Hence, all the forces along the direction of vehicle movement have been clarified, and the equation of motion is formed as

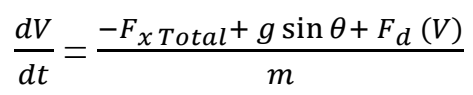

where $F_{x T o t a l}$ is the total force in $X$-direction acting on the vehicle body and $F_{d}(V)$ is the force due to drag.

\section{Drag Forces}

The drag force $F_{d}(V)$ in a vehicle is a combination of two types of resistance which are rolling resistance force and aerodynamic resistance force, such that $F_{d}=F_{a}+F_{r}$. The forces are dependent and act to limit the linear maximum speed of the vehicle. Aerodynamic force can be described mathematically as $F_{a}=\frac{1}{2} \rho A C_{d}\left(V^{2}\right)$, while $F_{r}$ as the resistance force acting on the wheel is given by $F_{r}=m g C_{r}(V)$, where $C_{r}$ is the rolling resistance coefficient, $A$ is the frontal area of the vehicle, $C_{d}$ is the aerodynamic drag coefficient, and $\rho$ is the density of air, which is $1.23 \mathrm{~m}^{3}$.

\section{Tyre Longitudinal Slip}

The longitudinal slip of the tyres is given as $\lambda_{i j}=\frac{V-i j R}{\max \left(V, \omega_{i j}\right)}$. To represent the characters of the tyres, the Pacejka Magic Formula tyre model proposed by [17]is used in this study. The mathematical equations as well as the parameters of the modelling can be seen in Aparow et al. [7].

\section{Engine Dynamic Model}

An internal combustion engine can be modelled as a second-order polynomial equation by referring to the engine torque curve characteristics. The relations between engine rpm and engine torque in the characteristic can be observed in the form of a polynomial equation such as Eq.(4), where rpm is engine angular speed in the form of revolutions per minute, while $\alpha, \beta$ and $\delta$ are the constant values for the equations. The engine rpm can be estimated by multiplying the wheel angular velocity of the driving axle with the current gear and final drive ratios. Thus the engine rpm can be written as $R P M=$ $\eta_{g} \eta_{f} \omega_{f}$

$$
T_{\max }=\alpha(R P M)^{2}+\beta(R P M)+\delta
$$

In the internal combustion engine, it is assumed that a time lag is experienced during the conversion of chemical energy to kinetic energy and also during the actuation of the throttle (which is actuated by a servo motor). The time lags are approximately 0.2 seconds, which is denoted by $\tau_{e s}$. By defining the actual amount of torque that is used as the function of torque maximum, $T_{M a x}$, and energy transfer coefficient, $\mu_{e}$, the equation $T_{e_{f i}}=\mu_{e} T_{M a x} \eta_{g} \eta_{f}$ can be utilised to state the relation between the drive 
torque and throttle setting, $\mu_{t}$, where $\mu_{e}=0.01 \mu_{t}-\tau_{e s} \mu_{e}$, while $\eta_{g}$ is the current gear ratio, $\eta_{f}$ is the final drive ratio and $\mu_{t}$ is the input throttle setting $(0-100 \%)$.

\section{Gearbox Model}

In modelling the gearbox, a shift logic system can be used to describe the automatic transmission gearbox. The shift logic is able to produce an approximate mapping that relates the threshold for changing the gear up or down as a function of wheel speed and throttle setting [14]. The simplified shift logic for five speed transmission can be seen in Figure 2.

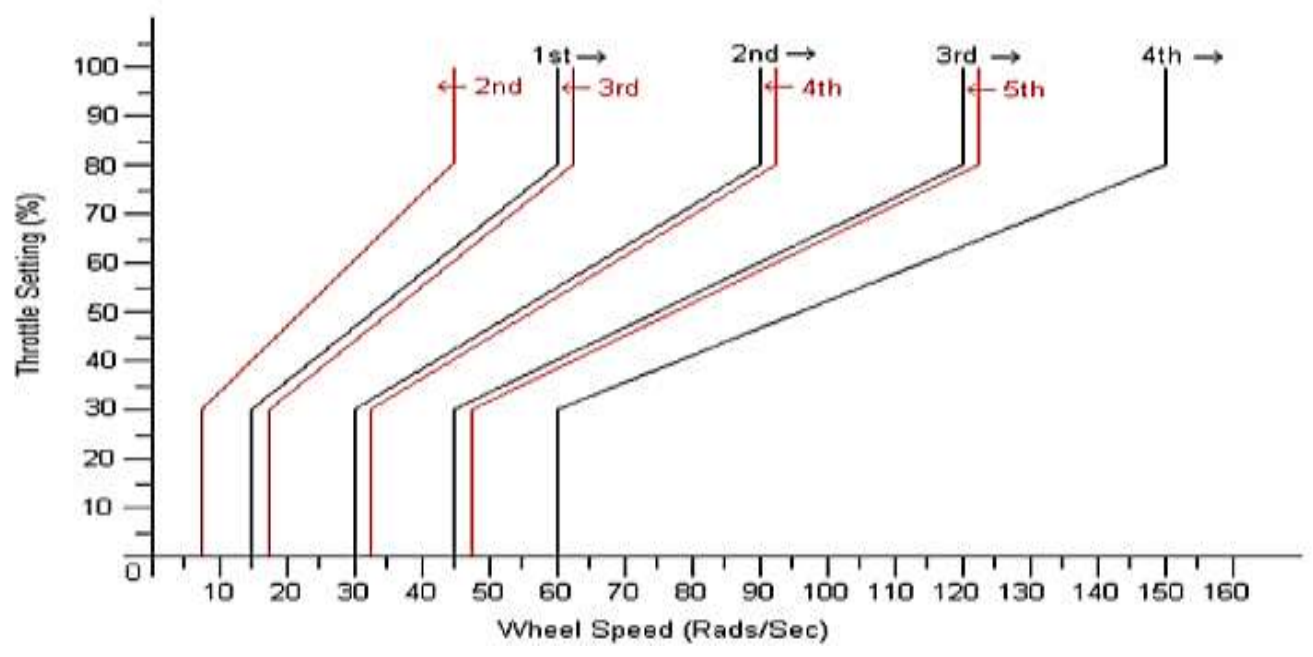

Figure 2. Automatic transmission gearbox shift logic [17].

\section{Brake System Model}

By referring to Imaduddin et al. [8], the pressure applied to the brake disc can be found as Eq.(5), Where $P_{b}$ is the brake pressure applied, $K_{c}$ is the simple pressure gain, $u_{b}$ is the brake setting and $\tau_{b s}$ is the brake lag. Considering $\vartheta$ as the simple pressure gain, the brake torque can be calculated as Eq.(6):

$$
\begin{gathered}
P_{b i j}=1.5 K_{c i j} u_{b i j}-\tau_{b s} \dot{P}_{b i j} \\
T_{b i j}=P_{b i j} K_{b i j} \min \left(1, \frac{\omega_{i j}}{\vartheta_{i j}}\right)
\end{gathered}
$$

\section{RESULTS AND DISCUSSION}

In order to verify the effectiveness of the vehicle longitudinal model, several experimental tests were performed using an instrumented experimental vehicle. In this validation study, a visual technique such as that utilised by Ahmad et al. [2] is employed. For validation purposes, a Malaysian national car, namely a Proton Iswara, was used. The vehicle is a hatchback equipped with a $1300 \mathrm{cc}$ engine capacity including with a five-speed forward-gear manual transmission as the power terrain systems. The experimental vehicle is equipped with a data acquisition system (DAS) in order to obtain the real vehicle reaction as to evaluate the vehicle's performance. The DAS uses 
several types of transducers, such as a wheel speed sensor to measure the angular velocity of the tyre, an infrared vehicle speed sensor to quantify the body longitudinal velocity, and a load cell to measure the distributed mass at each corner of the vehicle. The multi-channel Integrated Measurement and Control (IMC) $\mu$-MUSYCS system is used as the DAS system. The DAS system then is integrated with online FAMOS software for real-time data processing, display and data collection. The installation of the DAS and sensors in the experimental vehicle can be seen in Figure 3

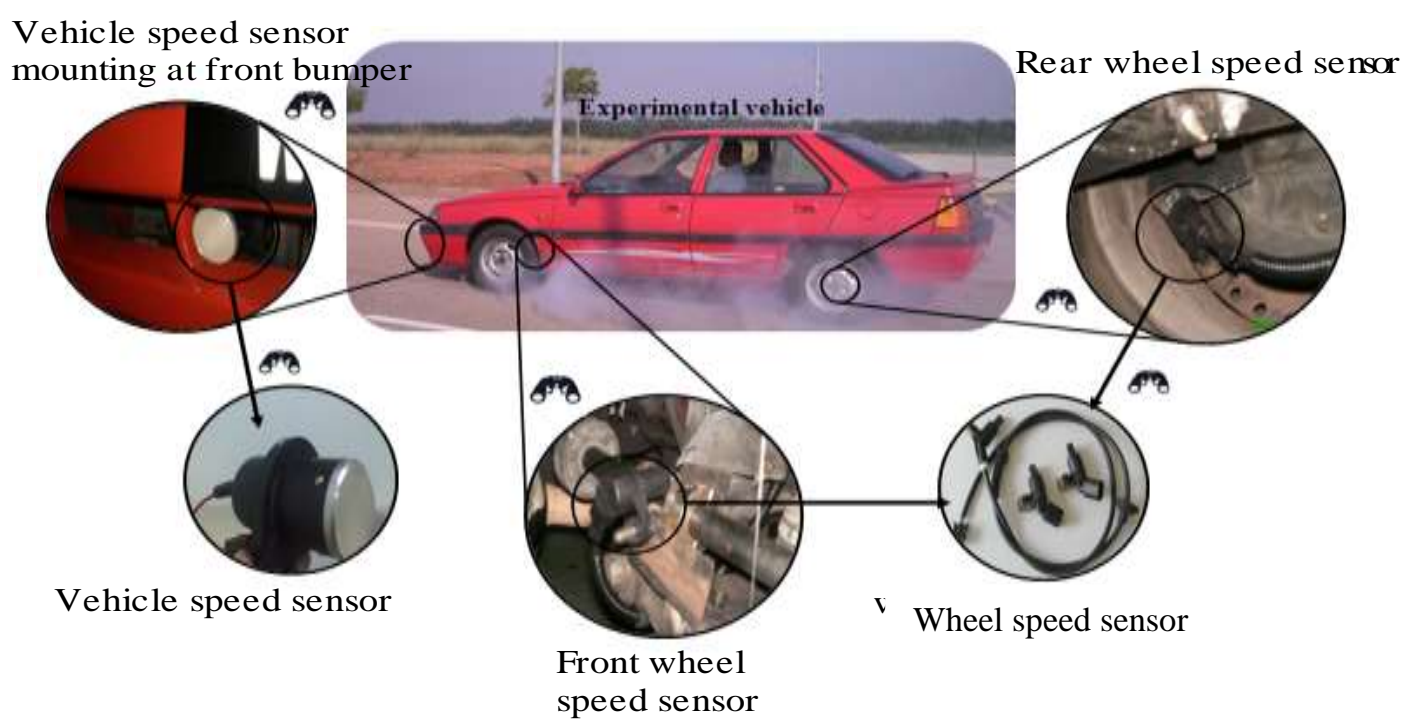

Figure 3. Experimental vehicle and instrumentations.

\section{Validation}

In the early stages of the validation, an experimental study to measure the location of $C G$ was conducted. The parameters that were observed are the position of $C G$ from the front axle, rear axle and to the ground. The next stage in this study was to obtain the engine torque curve of the real experimental vehicle via the engine dynamometer. The purpose of this experiment is to provide real data which will associate the engine dynamics with the model in the simulation. For validation purposes, handling test procedures, namely acceleration and then braking tests, were carried out to study the transient response of the vehicle. In this study, the test was conducted by accelerating the vehicle to a constant speed of $40 \mathrm{~km} \cdot \mathrm{h}^{-1}$ and the driver then applying the brake pedal hard enough to hold the pedal firmly until the vehicle stopped completely. The behaviours observed in this experiment are vehicle speed, wheel angular and linear speeds, and longitudinal slip occurring in each wheel. In order to strengthen the validity of the vehicle model, the same experiment was also conducted with a different speed of the vehicle, which was $60 \mathrm{~km} \cdot \mathrm{h}^{-1}$.

Position of CG Measurement: To determine the position of $C G$, which is located by the dimensions $C$ and $B$ in Figure 4, The vehicle needs to be in arbitration on the weighting scale and from the calculated moment the position of $C G$ or the distance of $C$ and $B$ can be obtained. Figure 8(a,b) shows the diagram of static loads on level ground and on a slope. Note that, $C G$ is the centre of gravity of the vehicle; $F_{z f}, F_{z r}, F_{z f l}$ and $F_{z r l}$ are the measured forces using a weighing scale at the front and rear axles respectively. The method for calculating the $C G$ is the same as used by Steve (2008). The position of 
the $C G$ from the front axle and rear axle is found as $1340 \mathrm{~mm}$ and $1040 \mathrm{~mm}$, respectively, while the height of the $C G$ from the ground is $600 \mathrm{~mm}$, as shown in Table 1.

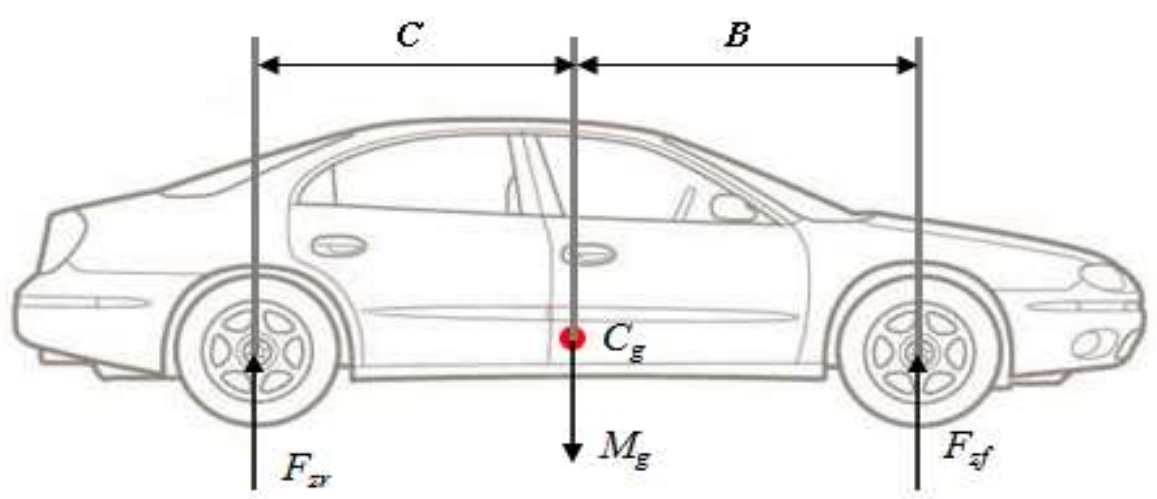

(a)



(b)

Figure 4. Vehicle on weighting scale (a) static loads on level ground; (b) Static loads on grades

Table 1. Proton Iswara general technical specifications.

\begin{tabular}{ll}
\hline Parameters & Value \\
\hline Vehicle mass & $920 \mathrm{~kg}$ \\
Wheel base & $2380 \mathrm{~mm}$ \\
Wheel track & $1340 \mathrm{~mm}$ \\
Wheel rolling radius & $220 \mathrm{~mm}$ \\
Front axle to $C G$ & $1340 \mathrm{~mm}$ \\
Rear axle to $C G$ & $1040 \mathrm{~mm}$ \\
Height $C G$ from ground & $600 \mathrm{~mm}$ \\
Gear ratios: & \\
$1^{\text {st }}$ & 3.363 \\
$2^{\text {nd }}$ & 1.947 \\
$3^{\text {rd }}$ & 1.285 \\
$4^{\text {th }}$ & 0.939 \\
$5^{\text {th }}$ & 0.777 \\
Final drive & 4.322 \\
\hline
\end{tabular}


Engine Dynamometer: An engine torque curve is needed to model the engine dynamic in the vehicle simulation. Therefore an engine dynamometer type Chassis DynoJet Mustang, which is available in the Engine Performance Testing Lab of Universiti Teknikal Malaysia Melaka, was utilised to obtain the actual torque produced by the engine versus rpm. A four-cylinder in-line 12-valve 4G13-type engine was used in the experimental vehicle. The observation results of the engine dyno are shown in Figure 5. In this study, experiments on the engine dyno were repeated three times to ensure the consistency of the data captured. Based on the Figure 5, the dashed line represents the final experimental data, while the solid line describes the basic fitting curve, which was developed to characterise a mathematical equation of the engine model. Equation (4) is employed to characterise the engine characteristics and is estimated as:

$$
T_{\max }=-0.43(R P M)^{2}+3.7(R P M)+5.3
$$

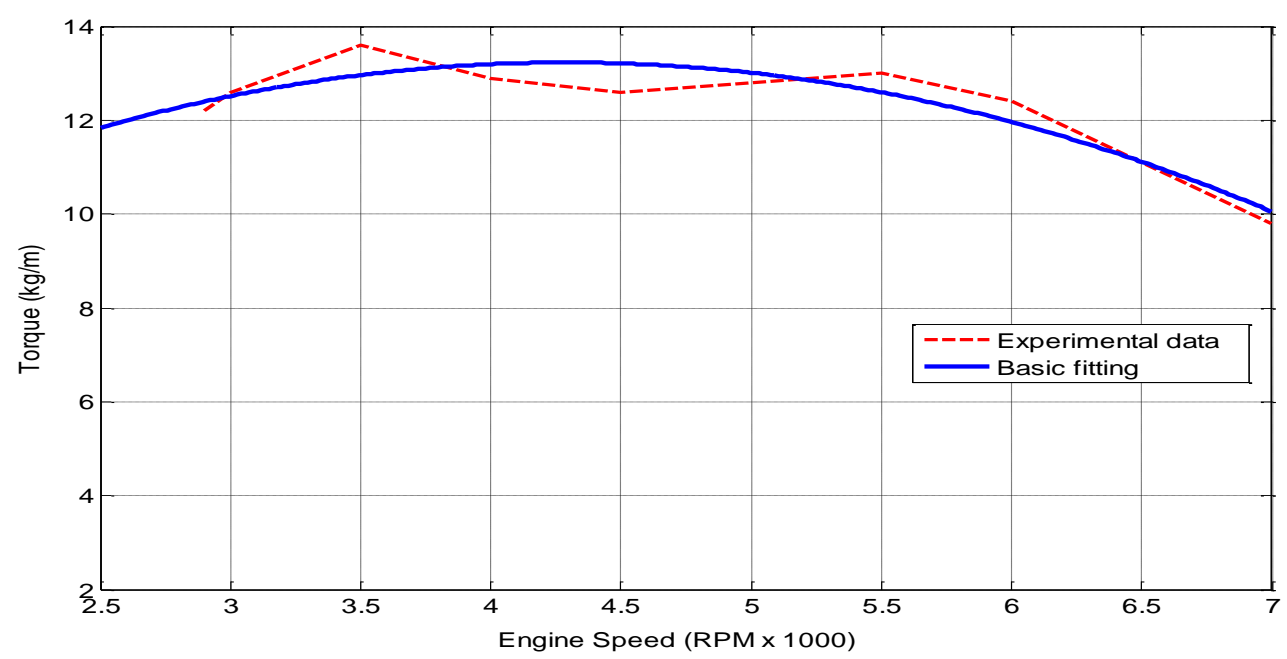

Figure 5. Engine dynamometer data.

\section{Validation Results}

Figures 6 to 7 show the results of the output variables obtained for $40 \mathrm{~km} \cdot \mathrm{h}^{-1}$ and 60 $\mathrm{km} \cdot \mathrm{h}^{-1}$ with 0 degree input steering for comparison. For validation purposes, all the experimental data are filtered using a low pass filter, filter order 8 and a pass band edge frequency of 30 to remove any unintended data. It should be noted that the measured vehicle speed from the speed sensor is used as the input of the simulation model. The tyre parameters used in the simulation model are shown in Table 1.

\section{Validation of Vehicle at $40 \mathrm{~km} \cdot \mathrm{h}^{-1}$ Constant Speeds}

The results of model validations for the acceleration and then braking test at $40 \mathrm{~km} \cdot \mathrm{h}^{-1}$ are shown in Figures $10(\mathrm{a}-\mathrm{j})$. In these figures, the red lines denote the responses of the experimental vehicle while the blue lines represent the responses of the vehicle simulation model. It can be seen that in the first phase, which is from $0-10$ seconds, the vehicle accelerates to a longitudinal speed of $40 \mathrm{~km} \cdot \mathrm{h}^{-1}$ and maintains the speed until the next 5 seconds. During transient period of 17 second, maximum brake is applied until the vehicle comes to a complete stop, which is represented by the second phase. 


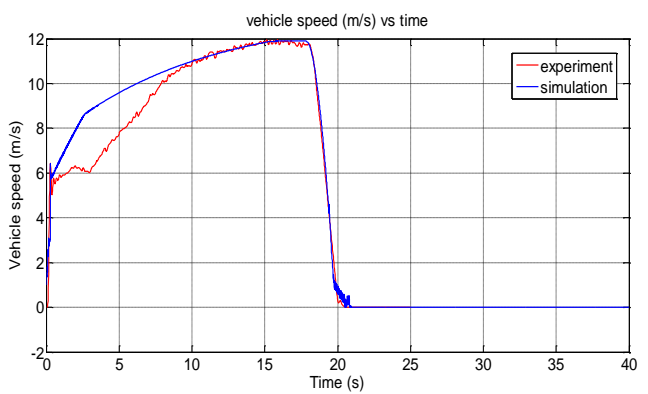

(a)

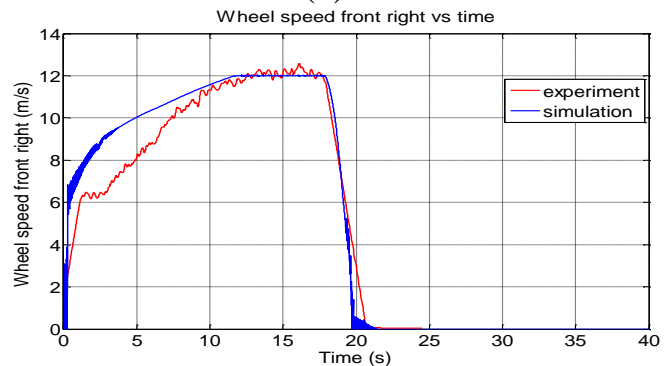

(c)

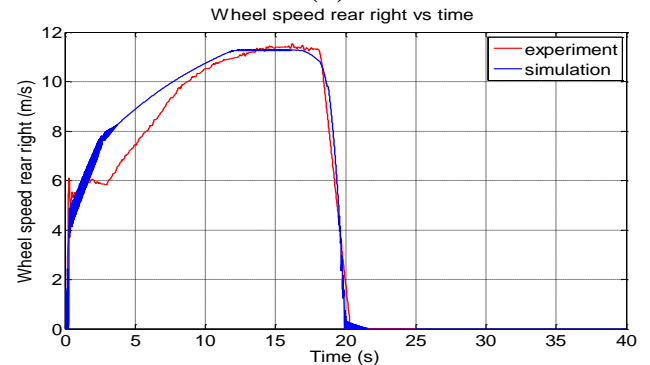

(e)

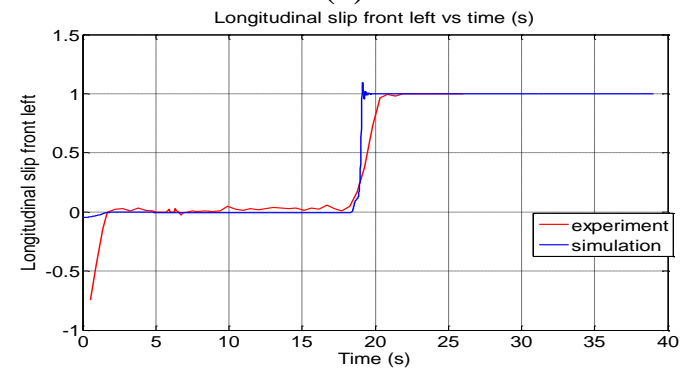

(g)



(i)

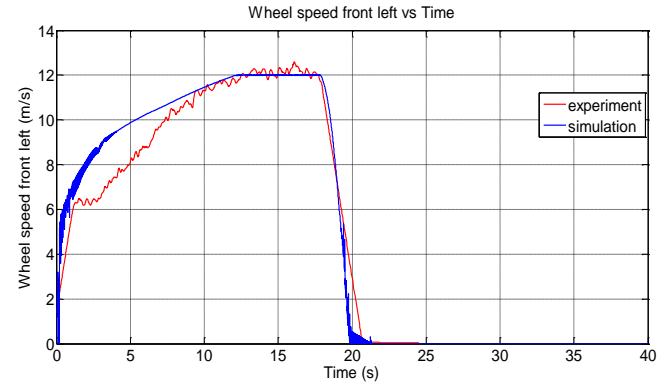

(b)



(d)

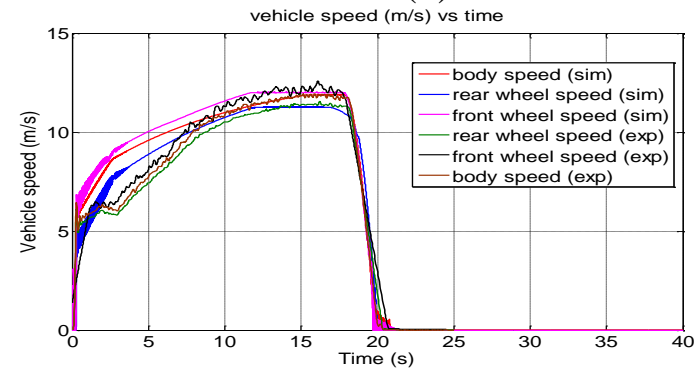

(f)

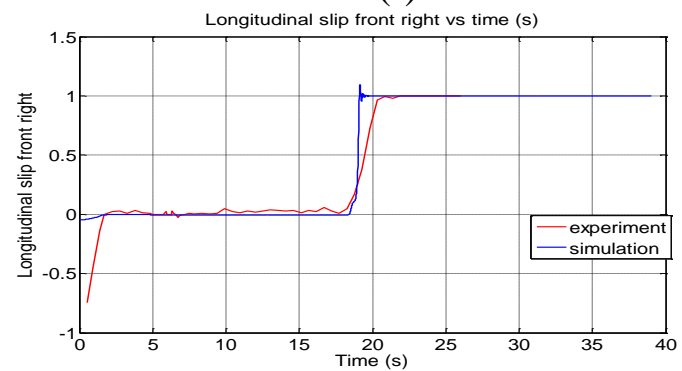

(h)

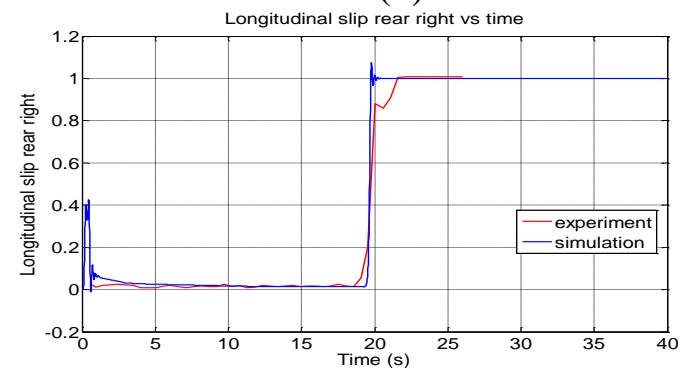

(j)

Figure 6. Validation result for the sudden braking test at $40 \mathrm{~km} \cdot \mathrm{h}^{-1}$ constant speed (a) Vehicle speed; (b) Wheel speed front left; (c) Wheel speed front right; (d) Wheel speed rear left; (e) Wheel speed rear right; (f) Comparison speed of body and wheels; (g) Longitudinal slip front left; (h) Longitudinal slip front right; (i) Longitudinal slip rear left; (j) Longitudinal slip rear right. 
From the observation, it can be noted that the trends between the simulation results and experimental data are almost similar with acceptable error. The small difference in magnitude between the simulation and experimental results is due to the fact that in the actual experiment it is indeed very hard for the driver to maintain the vehicle at a perfect speed compared with the result obtained in the simulation. Meanwhile, a profound mismatch is visible between the simulation and experiment around $t=2$ seconds until $t=10$ seconds, where the simulation response is slightly higher than the experimental response. The difference in the responses is due to the discontinuous gear shifting, which has a time delay, rpm drop and inconsistent clutch engage to the flywheel when the driver shifts the gear and disengages the clutch manually while shifting gears from one to another in the experimental vehicle. These conditions cause interruption in delivering engine torque to the wheel. Unlike the experimental vehicle, automatic transmission is utilised in the vehicle simulation. The automatic transmission works independently without frequent interruption, resulting in comfort and continuous gear shifting. Fundamentally, the movement of a vehicle, whether forward or backward, is caused by the rotation of all the tyres. Therefore, the behaviour of the vehicle in Figure 6(a) results from the rotation of all the vehicle's wheels as represented in Figures 6(b-e). From the observation, it can be seen that there are quite good comparisons during the initial transient phase as well as during the following steady state phase, but again there is disparity between time from $t=2$ seconds to $t=10$ seconds that have been discussed earlier. During the braking phase, the maximum brake force is applied at $t=17$ seconds to make the vehicle to stop immediately, hence the wheels are totally stopped 2 seconds later at $t=19$. According to [1], since the model was developed to examine the behaviour of the vehicle during braking, the small differences during the acceleration phase, i.e. at time $t=2$ seconds to $t=10$ seconds, can be ignored. According to Siegler and Crolla [17], engine torque is used to accelerate the vehicle only and the effect of the torque on the braking is very small and can be neglected. As the vehicle longitudinal model is developed based on front-wheel drive, the effect of the engine during initial start-up occurs in the front wheels only. Based on Figure 6(f), the vehicle stopped accelerating at $t=20$ seconds after brake input was applied at $t=17$ seconds. Unfortunately, the wheel starts to lock up at $t=19$ seconds and drags the vehicle until the vehicle comes to a complete stop, as shown in Figures 10(b-f). This causes the four wheels of the vehicle to undergo slip conditions on a normal road surface. Figures $10(\mathrm{~g}-\mathrm{j})$ show a validation of longitudinal slip that reaches +1 after $t=20$ seconds (after brake input is applied). This condition occurs once the wheel starts to lock up after the sudden brake input is applied and causes the wheels to skid and have no rotational motion due to wheel lock-up. The longitudinal slip responses of all the tires show satisfactory matching with only a small deviation in the transition area between transient and steady state phases. It can also be noted that the longitudinal slip responses of all the tyres in the experimental data are slightly different than the longitudinal slip data obtained from the simulation responses, particularly for the rear tyres. This is due to the fact that it is difficult for the driver to maintain a constant speed during manoeuvring. In simulation, it is also assumed that the vehicle is moving on a flat road during the manoeuvre. In fact, it was observed that the road profiles of the test field consist of an irregular surface. This can be another source of deviation on the longitudinal slip response of the tyres. 


\section{Validation of Vehicle model at $60 \mathrm{~km} \cdot \mathrm{h}^{-1}$ Constant Speeds}

To further investigates the validity of the vehicle model, another assessment was made using the same tests conditions, except that the vehicle was first accelerated to a constant speed of $60 \mathrm{~km} \cdot \mathrm{h}^{-1}$. Figures 7 shows the comparison results for the same output variables obtained under the condition of a 0 degree steering angle and a 60 $\mathrm{km} \cdot \mathrm{h}^{-1}$ longitudinal vehicle speed. In terms of vehicle longitudinal speed, the observations indicate that measurement data and the simulation results agree with a relatively good accuracy, as shown in Figure 7(a) but there is a difference in the first transient response which is between 0 to 5 seconds. From the figures, the response of the simulation shows that the vehicle takes around 5 seconds to achieve a constant speed of $60 \mathrm{~km} \cdot \mathrm{h}^{-1}$ from rest condition. Unfortunately, the experimental response shows that the vehicle can speed up from rest condition to a constant speed of $60 \mathrm{~km} \cdot \mathrm{h}^{-1}$ within 1 second. As a discussion of this problem, it is believed that the disparity is caused by an error when activating the sensory device. During the experimental validation of this condition, the driver was late to activate the sensor device. All the sensors were then activated when the speed of the vehicle was accelerating to a constant speed of $60 \mathrm{~km} \cdot \mathrm{h}^{-}$ ${ }^{1}$ so that the step response at 5 seconds of the experimental data indicates that the sensor is firstly switch on. Actually, there are several other experiments which show that the vehicle needs around 5 to 6 seconds to reach $60 \mathrm{~km} \cdot \mathrm{h}^{-1}$ from rest condition.

In terms of wheel linear speed, the results indicate that the measurement data and the simulation results agree with a relatively good accuracy, as shown in Figures 11(be), except for the first 5 seconds. Almost similar to the validation results obtained from the first test, the linear speed responses in the experimental data are higher than the linear speed data obtained from the simulation, particularly for all the tyres. Again, this is due to the difficulty of the driver to maintain a constant speed during the manoeuvre. The assumption in simulation model that the vehicle is moving on a flat road during the manoeuvre is also very difficult to realise in practice. In fact, road irregularities of the test field may cause a change in tyre properties during the vehicle handling test. The assumption of neglecting the steering inertia has the possibility of lowering the magnitude of tyre linear speed in simulation results compared with the measured data. In the first 5 seconds of the simulation model, the responses indicate that the vehicle tried to accelerate as fast as possible to reach the desired constant speed by supplying height throttle torque to the wheel. As a result, height wheel angular velocity is produced and resulting spinning of the tyres occurs, as illustrated in the longitudinal slip figures (Figures $7(\mathrm{~g}-\mathrm{j})$ ). Apart from that, comparisons of the body longitudinal speed and wheel linear speed are made to observe the behaviour of the vehicle in a sudden braking manoeuvre. The comparison of those velocities is presented in Figure 7(f) by comparing all the simulation results and experimental responses. From the figure, it can be explained that the front wheel velocity is slightly higher than the velocity of the vehicle body, while the rear wheel velocity is lower. Since the vehicle is front-wheel drive, there is no doubt that this condition could have occurred because the vehicle movement is triggered by the front wheels - in fact there is no input drive torque in the rear wheels. In other words, during heavy acceleration, weight is shifted from the front to the rear and causes increased traction in the rear wheels at the expense of the front driving wheels; consequently, longitudinal speed is raised in the front tyres as a result of wheel spinning [17]. 


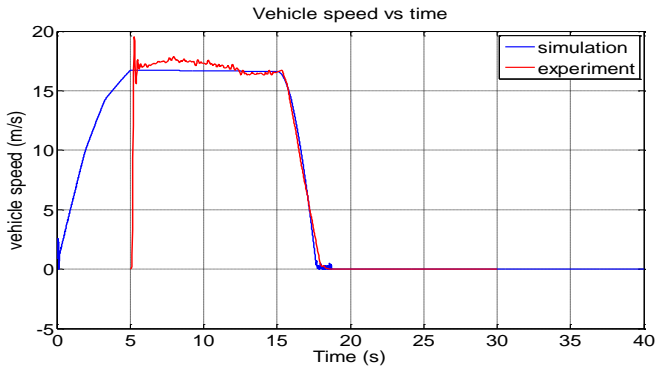

(a)

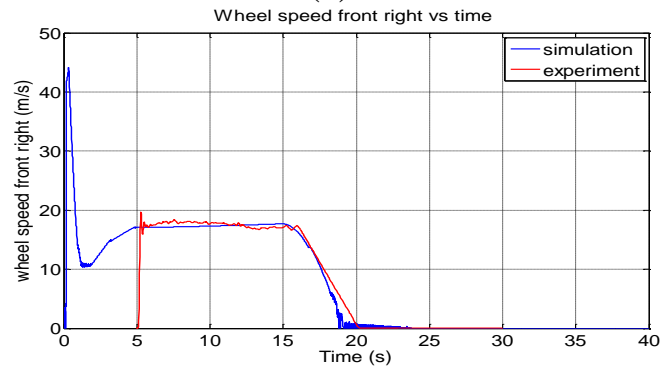

(c)



(e)

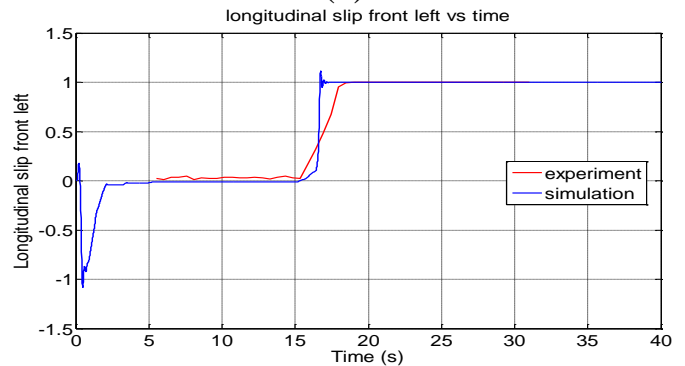

(g)

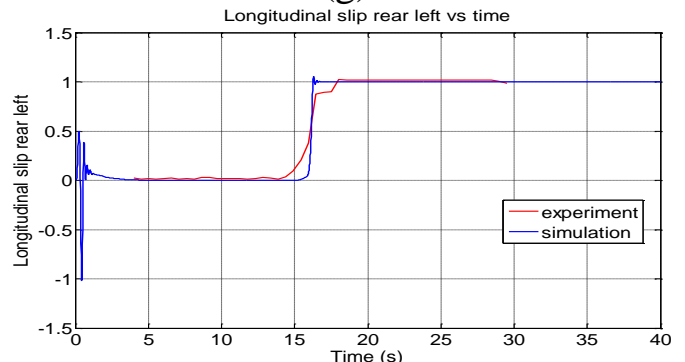

(i)



(b)

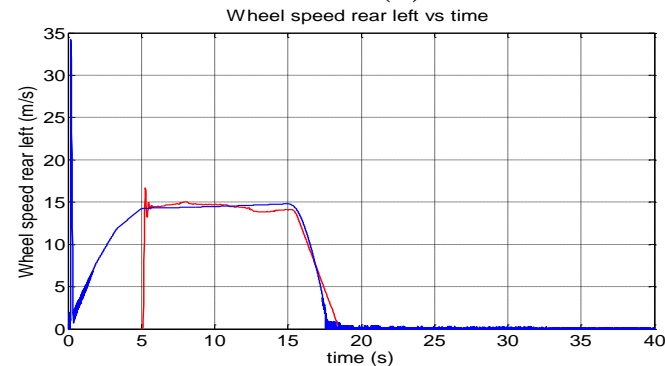

(d)

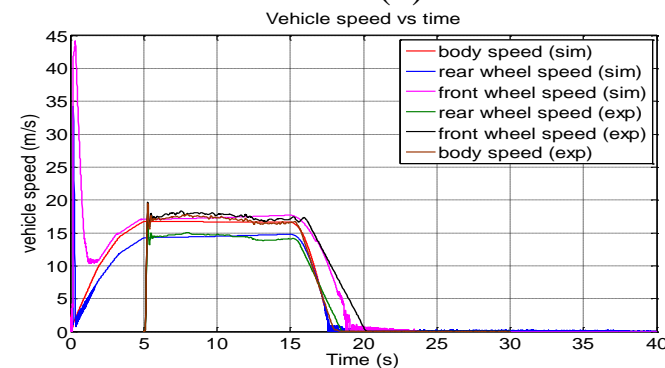

(f)

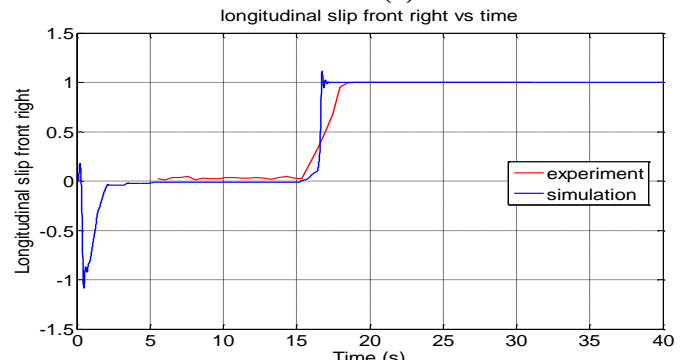

(h)



(j)

Figure 7. Validation result for a sudden braking test at a constant speed of $60 \mathrm{~km} \cdot \mathrm{h}^{-1}$ (a) Vehicle longitudinal speed; (b) Wheel speed front left; (c) Wheel speed front right; (d) Wheel speed rear left; (e) Wheel speed rear right; (f) Comparison speed of body and wheels; (g) Longitudinal slip front left; (h) Longitudinal slip front right; (i) Longitudinal slip rear left; (j) Longitudinal slip rear right 


\section{CONCLUSIONS}

It can be concluded that the trends between simulation results and experimental data show good agreement with acceptable errors for our purposes. The deviations in the responses are believed to be caused by various simplified modelling assumptions, particularly the consideration of negligible contributions due to constant forward speed, absence of wheel hop, and linear suspension properties. The errors could be significantly reduced by fine-tuning both vehicle and tyre parameters. Hence, the vehicle longitudinal model is able to represent behaviour that is similar to a real vehicle. Finally, it is suggested that this model can be employed as an effective tool in future studies, such as to investigate and prototype the performance of an anti-lock braking system design before running tests upon a real vehicle.

\section{ACKNOWLEDGEMENT}

This work is supported by the Malaysia-Japan International Institute of Technology (MJIIT), Universiti Teknologi Malaysia through its scholarship and financial support, and Universiti Teknikal Malaysia Melaka (UTeM) through the short-term grant research project (PJP) entitled "Safety and Stability Enhancement of Automotive Vehicle Using ABS with Electronic Wedge Brake Mechanism", project no. PJP/2011/FKM(6B)/S00860 led by Fauzi Ahmad at the Universiti Teknikal Malaysia Melaka. This financial support is gratefully acknowledged.

\section{REFERENCES}

[1] Duwig C, Stankovic D, Fuchs L, Li G, Gutmark E. Experimental and numerical study of flameless combustion in a model gas turbine combustor. Combustion Science and Technology. 2007;180:279-95.

[2] Ahmad F, Hudha K, Imaduddin F, Jamaluddin H. Modelling, validation and adaptive PID control with pitch moment rejection of active suspension system for reducing unwanted vehicle motion in longitudinal direction. International Journal of Vehicle Systems Modelling and Testing. 2010;5:312-46.

[3] Freeman J, Watson G, Papelis Y, Lin T, Tayyab A, Romano R, et al. The Iowa driving simulator: An implementation and application overview. SAE Technical Paper; 1995.

[4] Katrin B, Elisabeth U, Erik G S, Urban B. On the ability of the 802.11 p MAC method and STDMA to support real-time vehicle-to-vehicle communication. EURASIP Journal on Wireless Communications and Networking. 2009;2009.

[5] Mousseau R, Markale G. Obstacle impact simulation of an ATV using an efficient tire model. Tire Science and Technology. 2003;31:248-69.

[6] Hudha K. Non-parametric modeling and modified hybrid skyhook groundhook control of magnetorheological dampers for automotive suspension system: Universiti Teknologi Malaysia, Faculty of Mechanical Engineering; 2005.

[7] Aparow VR, Ahmad F, Hudha K, Jamaluddin H. Modelling and PID control of antilock braking system with wheel slip reduction to improve braking performance. International Journal of Vehicle Safety. 2013;6:265-96.

[8] Imaduddin F, Hudha K, Mohammad JI, Jamaluddin H. Simulation and experimental investigation on adaptive multi-order proportional-integral control for pneumatically actuated active suspension system using knowledge-based 
fuzzy. International Journal of Modelling, Identification and Control. 2011;14:73-92.

[9] Ekoru JE, Pedro JO. Intelligent Feedback Linearizationbased Control of HalfCar Active Suspension Systems. Proceedings of the fifth IASTED Africa International Conference on Modelling and Simulation (AfricaMS 2012), Gaborone, Botswana2012. p. 161-8.

[10] Hudha K, Kadir ZA, Said MR, Jamaluddin H. Modelling, validation and roll moment rejection control of pneumatically actuated active roll control for improving vehicle lateral dynamics performance. International Journal of Engineering Systems Modelling and Simulation. 2009;1:122-36.

[11] Kadir Z, Hudha K, Ahmad F, Abdullah MF, Norwazan A, Mohd Fazli M, et al. Verification of 14DOF full vehicle model based on steering wheel input. Applied Mechanics and Materials. 2012;165:109-13.

[12] El Majdoub K, Giri F, Ouadi H, Dugard L, Chaoui FZ. Vehicle longitudinal motion modeling for nonlinear control. Control Engineering Practice. 2012;20:69-81.

[13] Short M, Pont MJ. Assessment of high-integrity embedded automotive control systems using hardware in the loop simulation. Journal of Systems and Software. 2008;81:1163-83.

[14] Short M, Pont MJ, Huang Q. Simulation of vehicle longitudinal dynamics. UK: Safety and Realiability of Distributed Embedded Systems Embedded Systems Laboratory University Leicester. 2004.

[15] Huang X, Wang J. Longitudinal motion based lightweight vehicle payload parameter real-time estimations. Journal of Dynamic Systems, Measurement, and Control. 2013;135:011013.

[16] Bakker E, Pacejka HB, Lidner L. A new tire model with an application in vehicle dynamics studies. SAE Technical Paper; 1989.

[17] Siegler B, Crolla D. Lap time simulation for racing car design. SAE Technical Paper; 2002. 